\title{
Simplify your Endodontics with Single File Systems- Case Reports
}

\author{
AnilDhingra ${ }^{1}$, PunitSrivastava $^{2}$, DiptiChadda $^{3}$, SatyabratBanerjee $^{4}$ \\ ${ }^{1}$ Professor \& HOD, MDS, , D.J. College of Dental Sciences \& Research, Department of Conservative Dentistry \\ \&Endodontics, Modinagar (U.P.), India \\ ${ }^{2,3}$ P.G. Student, BDS, D.J.College of Dental Sciences \& Research, Department of Conservative Dentistry \\ \&Endodontics, ,Modinagar (U.P.), India \\ ${ }^{4}$ Senior Lecturer, D.J.College of Dental Sciences \& Research, Department of Conservative Dentistry \\ \&Endodontics, Modinagar (U.P.), India
}

\begin{abstract}
A series of Case reports are presented using Single file system namely WaveOne Reciprocating System and OneShape Single file rotary system.

Clinical relevance:Nickel titanium rotary shaping files fundamentally changed everything in endodontics; conceptually, procedurally and economically as well. NiTi rotary files made root canal therapy more accessible and kind of fun for dentists, and dental companies were loved for making files that cost four times more than stainless steel hand files but delivered a ten times improvement. There is no need for disinfecting, cleaning, sterilizing and organizing the NiTi files, provide optimal cutting efficiency along with better control of file breakageand thus increased patient safety and no risk for cross contamination.
\end{abstract}

Key Words: WaveOne Reciprocating motion, OneShape Rotary motion, Pathfiles.

\section{Introduction}

Traditionally, the shaping of root canals was achieved by the use of stainless steel hand files. However, techniques using stainless steel hand files have several drawbacks (1).They require the use of numerous hand files and drills to adequately prepare the canals (Schilder 1947).Hand instrumentation with stainless steel files is time consuming (Ferraz et al. 2001).The stainless steel hand instrumentation techniques have an increased incidence of canal transportation (Kuhn et al. 1997, Reddy and Hicks 1998, Ferraz et al. 2001, Pettiette et al. 2001).Finally, from a clinical standpoint, the use of hand instruments in narrow canals can be very frustrating especially in teeth with difficult access.

Nickel titanium rotary shaping files fundamentally changed everything in endodontics; conceptually, procedurally and economically as well. NiTi rotary files made root canal therapy more accessible and kind of fun for dentists, and dental companies were loved for making files that cost four times more than SS hand files but delivered a ten times improvement. (2)

Recent advances for endodontic canal preparation have focused on the concept "Less is More". (3)

Thus a single-file technique has developed for shaping the vast majority of canals, regardless of their length, diameter, or curvature. (3)

\subsection{Case No-1: Management of Radix entomolaris with Wave one single file reciprocating system}

A 28 year old female patient referred to Department of Conservative dentistry\&Endodontics complaining of pain in lower right back region of mouth.On examination, tooth no 46 was previously restored with silver amalgam and was tender on percussion,

Radiographic examination showed deep amalgam restoration and secondary caries approaching the mesial and distal pulp horn with no periapical radiolucency (Fig 1).

A clinical diagnosis of Irreversible pulpitis with acute apical periodontitis was made and root canal therapy was planned. 


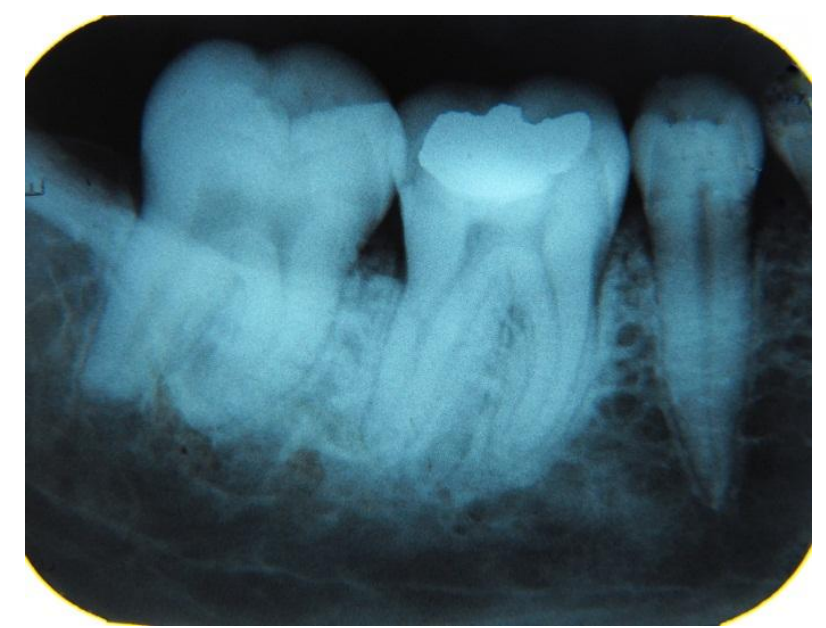

Figure 1: (pre-operative radiograph of tooth no 46)

Treatment procedure:Root canal treatment started under routine rubber dam isolation(Hygienic Dental dam, Coltene/WhaledentInc), and straight line access wasobtained Using No. 4 round bur(DentsplyMaillefer), Endo $\mathrm{Z}$ bur (DentsplyMaillefer) and four orifices were located (Fig 2). Working length determination done with radiograph (Fig 3) then glide path was achieved with pathfiles(DentsplyMaillefer) in rotary motion. Biomechanical preparation was completed done using WaveOne Primary reciprocating file (DentsplyMaillefer)and canal irrigation was done using continuous irrigation with $5.25 \%$ sodium hypochlorite.Master cone selection (Fig 4) and obturation done using AH-Plus as a sealer then post-endodontic restoration done using composite(Te-econom-plus, Ivoclar)(Fig 5).

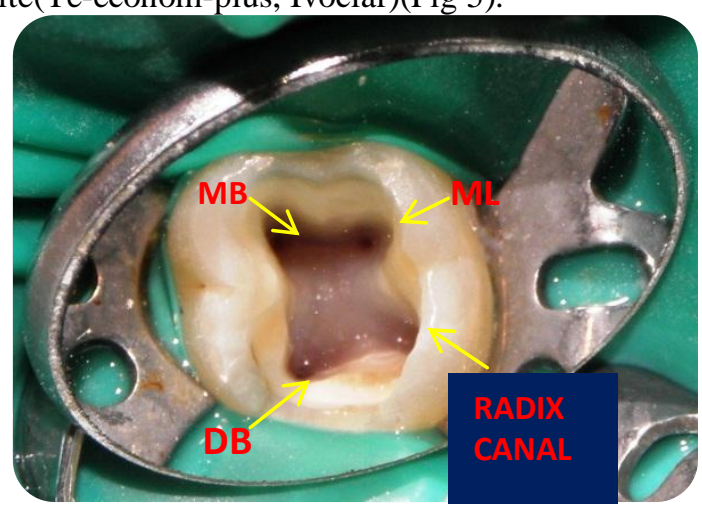

Figure 2:(upon access pulp chamber revealed 4 separate root canal orifices)

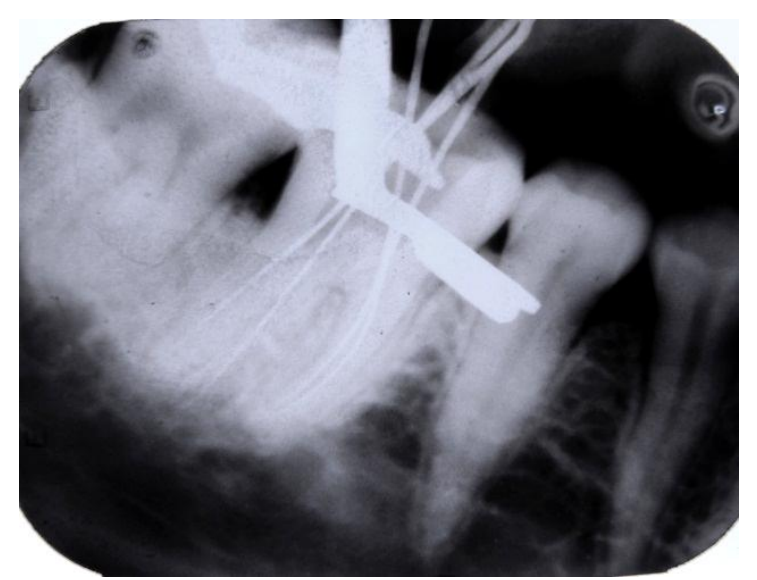

Figure 3 :(radiograph of working length determination tooth no 46) 


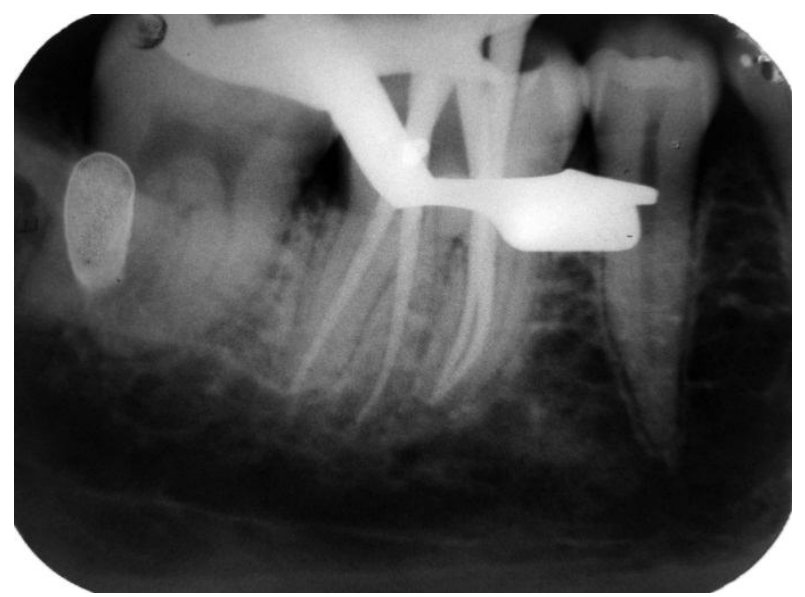

figure 4:(master cone selection done)

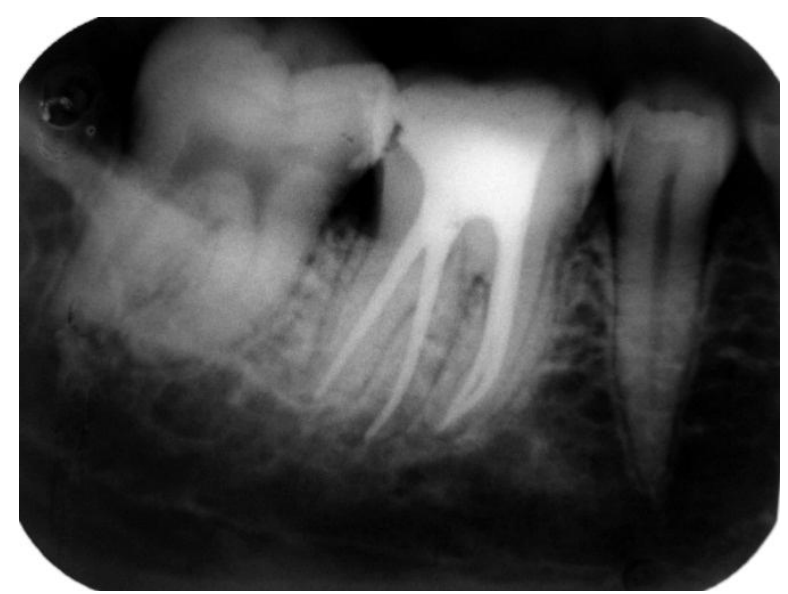

figure 5:(obturation done using monocone technique and ah plus as sealer and post endodontic restoration done with composite)

\subsection{Case No-2 :Single visit endodontics for lower left $1^{\text {st }}$ molar withWave Onesingle file reciprocating system}

A 35 year old male patient referred to Department of Conservative dentistry \&Endodontics complaining of pain in lower left back region of mouth. On examination, tooth no 36 was previously restored with temporary restoration and was tender on percussion,

Radiographical examination showed faulty restoration which was involving whole plulp chamber.No periapical radiolucency evident (Fig 6).

A clinical diagnosis of Irreversible pulpitis was made and root canal therapy was planned.

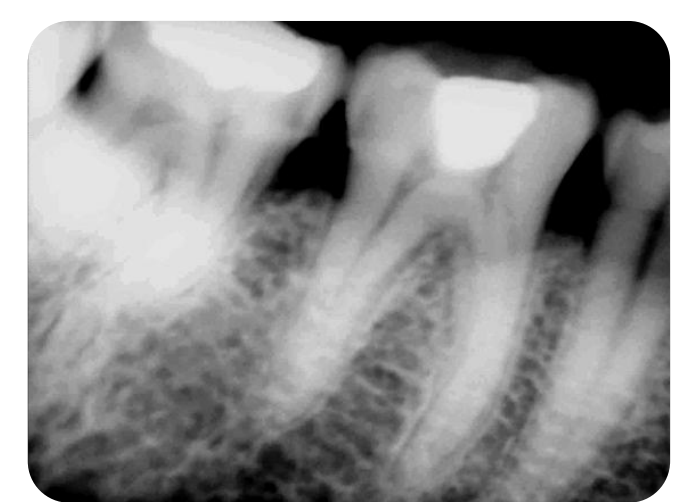

figure 6: (pre-operative radiograph of tooth no 36)

Treatment procedure: Root canal treatment started under routine rubber dam isolation(Hygienic Dental dam, Coltene/WhaledentInc), and straight line access was obtained Using No. 4 round bur (Dentsply,Maillefer), Endo Z bur (Dentsply,Maillefer) and four orifices were located (Fig 7) along with Working length determination 
with radiograph (Fig 8). Then glide path was achieved with pathfiles(Dentsply,Maillefer) in rotary motion. Biomechanical preparation was completed using WaveOne Primary reciprocating file (Dentsply, Maillefer) and canal irrigation was done using continuous irrigation with $5.25 \%$ sodium hypochlorite. Master cone selection done and obturation completed using AH-Plus as a sealer then post-endodontic restoration done using composite(Te-econom-plus, Ivoclar) (Fig 9 and 10).

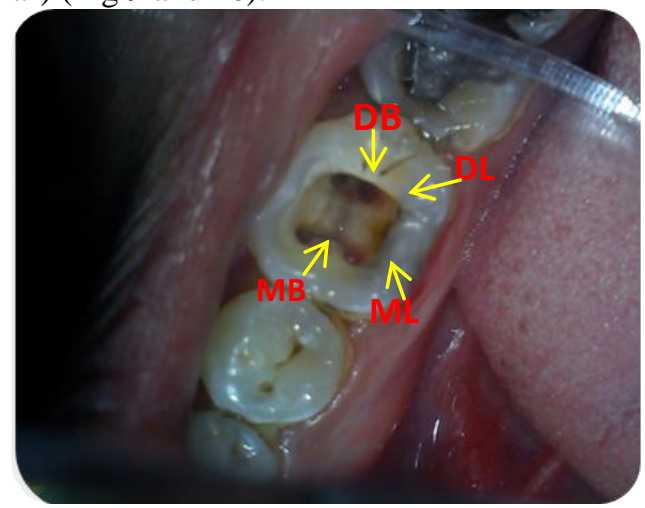

Figure 7:(upon access pulp chamber revealed 4separate root canal orifices)

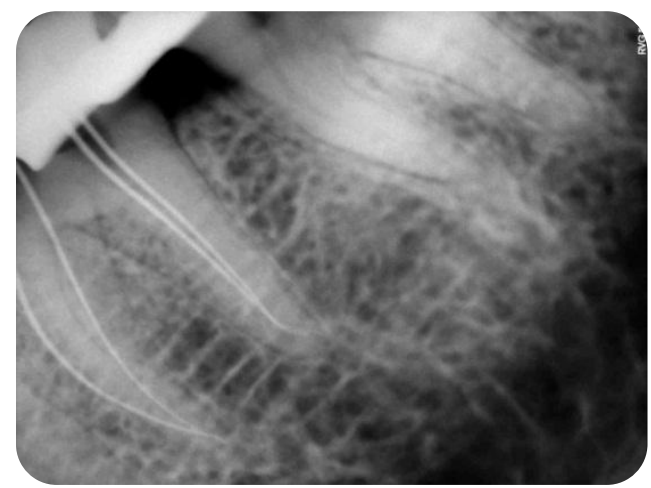

Figure 8:(radiograph of workinglength determination tooth no 36)

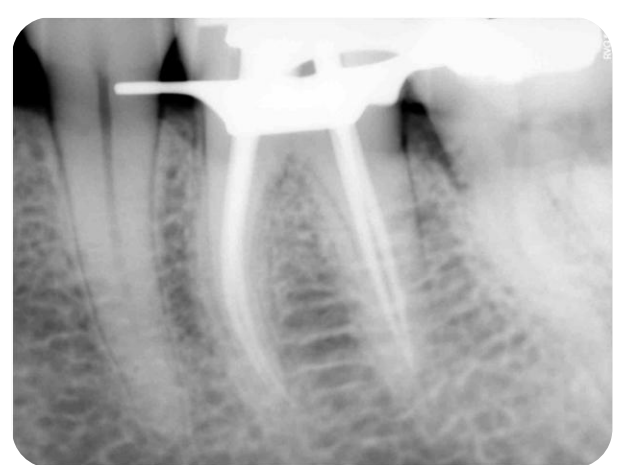

Figure 9:(master cone selection done)

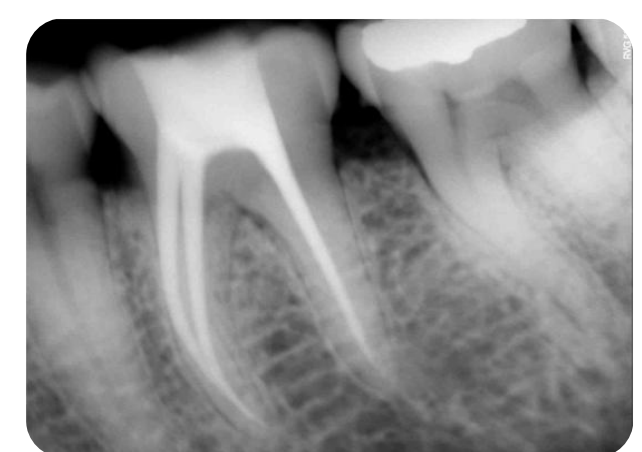

Figure 10:(obturation done using monocone technique and ah plus as sealer and post endodontic restoration done with composite) 


\subsection{Case No-3: Single visit endodontics for Maxillary left $1^{\text {st }}$ Molar with One-Shape single filerotary system}

A 39 year old male patient referred to Department of Conservative dentistry \&Endodontics complaining of pain in upper left back region of mouth. On examination, tooth no 26 was carious and was tender on percussion,

Radiographical examination caries involving plulpal floor no periapical radiolucency (Fig 11).

A clinical diagnosis of Irreversible pulpitis was made and root canal therapy was planned.

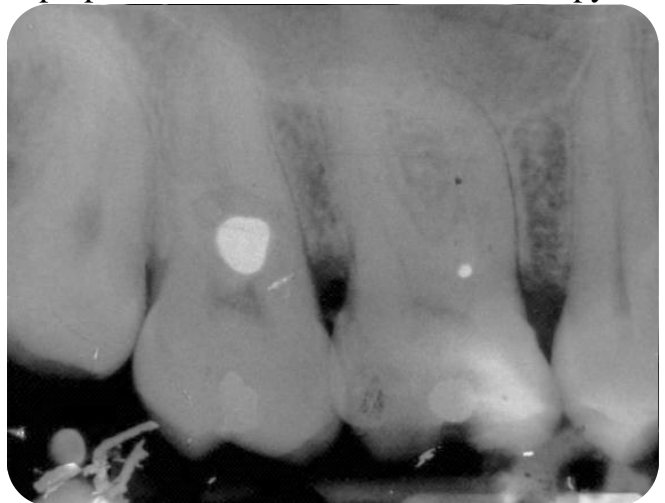

Figure 11: (pre-operative radiograph of tooth no 26)

Treatment procedure: Root canal treatment started under routine rubber dam isolation(Hygienic Dental dam, Coltene/WhaledentInc), and straight line access was obtained Using No. 4 round bur (Dentsply,Maillefer), Endo Z bur (Dentsply,Maillefer) and four orifices were located along with Working length determination with radiograph (Fig 12 and 13) then glide path was achieved with pathfiles(Dentsply,Maillefer) in rotary motion. Biomechanical preparation was completed done using Oneshape rotary file (MicroMega) and canal irrigation was done using continuous irrigation with 5.25\% sodium hypochlorite.Master cone selection done and obturation completed using AH-Plus as a sealer then post-endodontic restoration done using composite(Teeconom-plus, Ivoclar) (Fig 14 and 15).

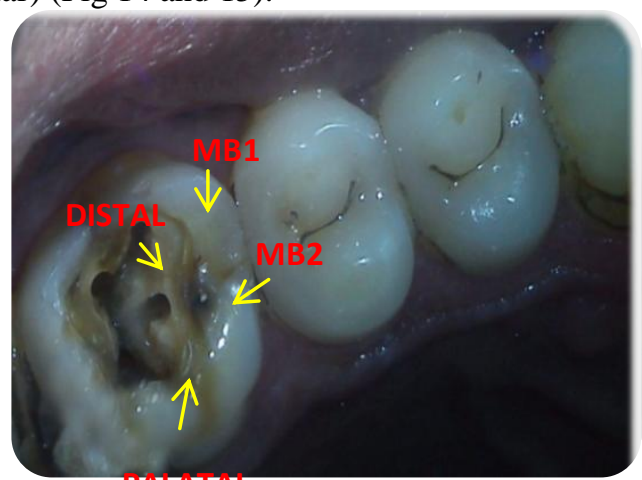

Figure 12:(upon access putp chatamber revealed 4 separate root canal orifices)

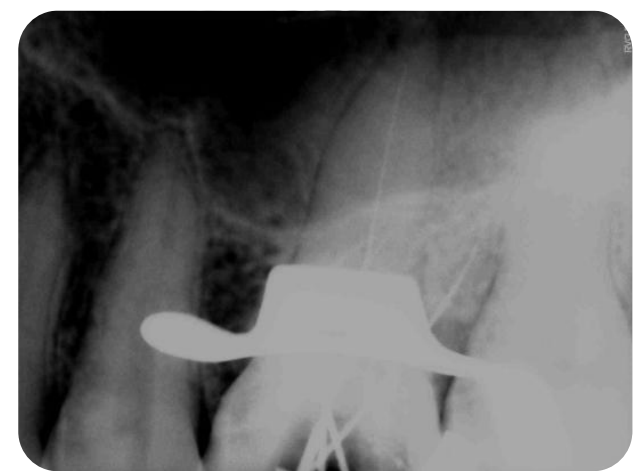

Figure 13 (radiograph of workinglength determination tooth no 26) 


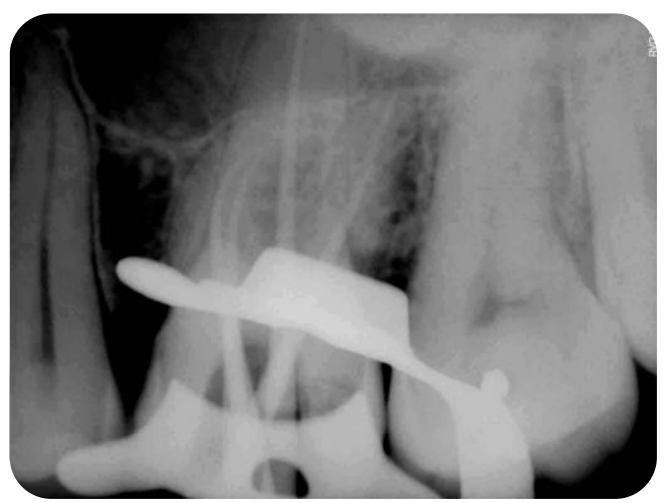

figure 14:(master cone selection done)

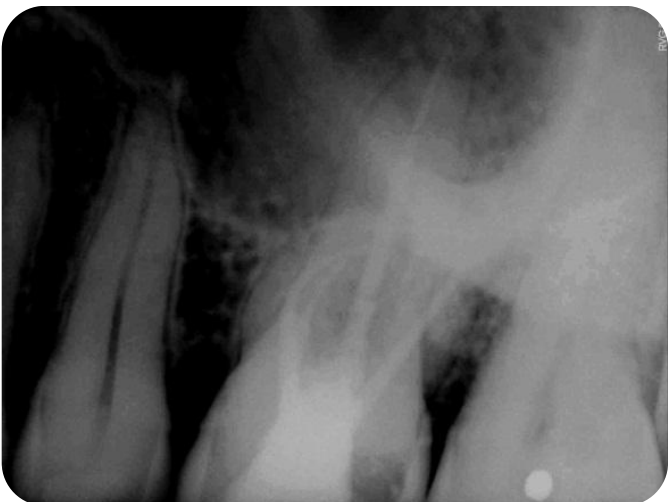

Figure 15: (obturation done using monocone technique and ah plus as sealer and post endodontic restoration done with composite)

\subsection{Case No-4: Management of mandibular right $1^{\text {st }}$ molar with 4 rootswith One shape single file rotary system}

A 25 year old male patient referred to Department of Conservative dentistry \&Endodontics complaining of food lodgement and low pain in lower right back region of mouth. On examination, tooth no 46 was grossly carious and was tender on percussion,

Radiographical examination caries involving plulpal floor no periapical radiolucency (Fig 16).

A clinical diagnosis of Irreversible pulpitis with acute apical periodontitis was made and root canal therapy was planned.

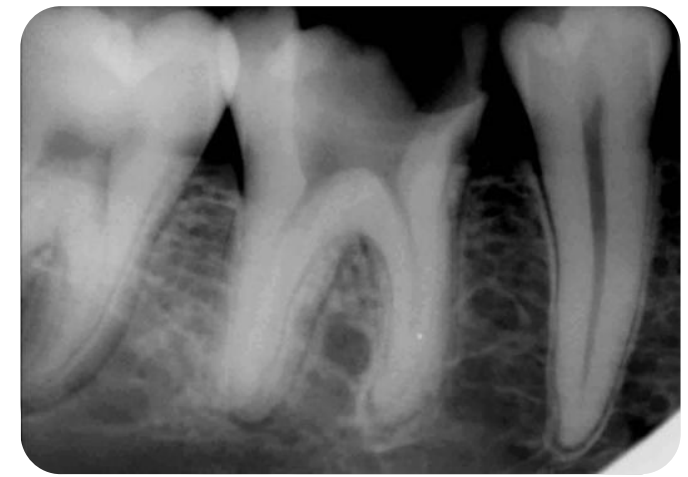

Figure 16:(pre-operative radiograph of tooth no 46)

Treatment procedure: Root canal treatment started under routine rubber dam isolation(Hygienic Dental dam, Coltene/WhaledentInc), and straight line access was obtained Using No. 4 round bur (Dentsply,Maillefer), Endo Z bur (Dentsply,Maillefer) and four orifices were located along with Working length determination with radiograph (Fig 17) then glide path was achieved with pathfiles(Dentsply,Maillefer) in rotary motion. Biomechanical preparation was completed done using Oneshape rotary file (MicroMega) and canal irrigation was done using continuous irrigation with $5.25 \%$ sodium hypochlorite. Master cone selection done and 
obturation completed using Zinc-oxide eugenol a sealer then post-endodontic restoration done using composite(Teeconom-plus, Ivoclar) (Fig 18 and 19).

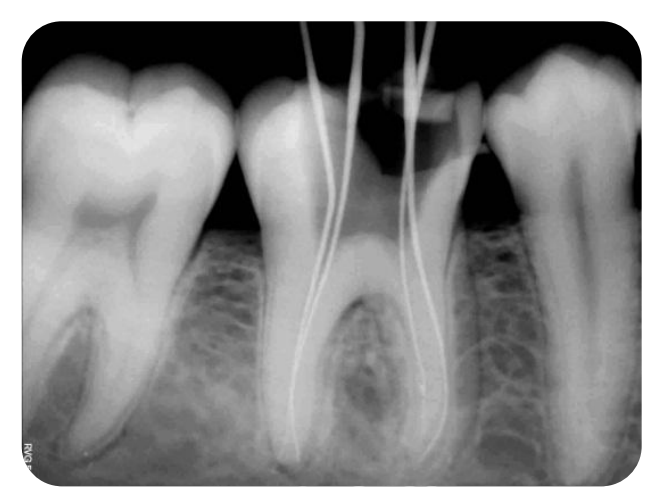

Figure 17:(radiograph of working length determination tooth no 26)

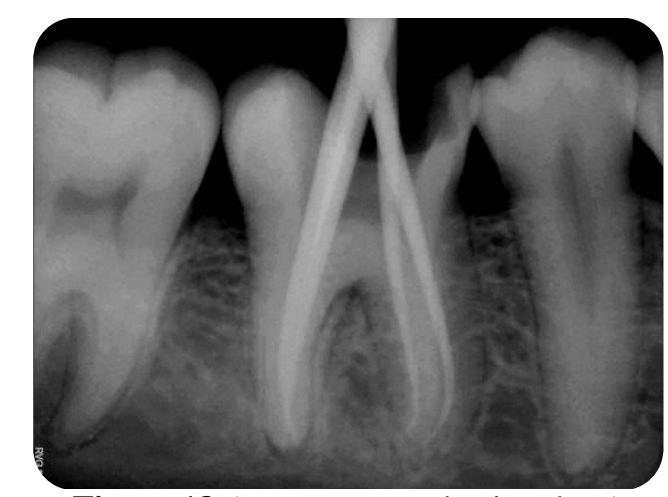

Figure 18:(master cone selection done)

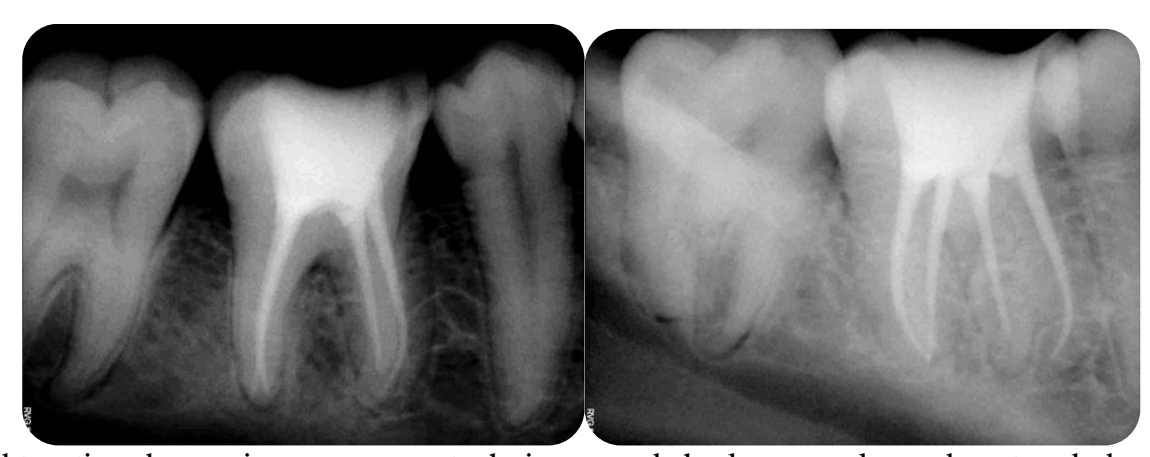

Figure 19:(obturation done using monocone technique and ah plus as sealer and post endodontic restoration done with composite)

\section{Discussion}

By far, the greatest number of commercially available files utilized to shape root canals are manufactured from NiTi and are mechanically driven in continuous rotation. (3)

On the other hand, reciprocation, defined as any repetitive back-and-forth motion, has been clinically utilized to drive stainless steel files since1958. [3]

The new WaveOneNiTi single-file system has been recently introduced by DentsplyMaillefer. The system is designed to be used with a dedicated reciprocating motion motor. [4]

It consists of 3 single-use files: small (ISO 21 tip and 6\% taper) for fine canals; primary (ISO 25 tip and 8\% taper) for the majority of canals; and large (ISO 40 and $8 \%$ taper) for large canals. The files are manufactured with M-Wire NiTi alloy. [4]

Whereas, One Shape file by MicroMega completes canal shaping with only one single file in continuous rotation. [5] It is 'Unique', 'Sterile', 'Economic' and 'Innovative' in nature. [5] One Shape ${ }^{\circledR}$ allows for curved canal negotiation with an instrumental and easy dynamic. Its non-working (safety) tip ensures an effective apical progression avoiding obstructions which are often preceded by instrument separation. [5] Quality root canal shaping with one single instrument with remarkable design. There is no need to buy an 
additional specific motor. You can use your traditional endohandpiece placed on your unit or any other endodontic motor with continuous rotation. [6]. It is the one and only one NiTi instrument in continuous rotation for quality root canal reparation $6 \%$ taper, $400 \mathrm{RPM}, 4 \mathrm{~N} / \mathrm{Cm} 2$ torque. [7]

When shaping canals, it should be appreciated that there are both advantages and disadvantages associated with utilizing continuous rotating vs a reciprocating movement. [3]

Compared to reciprocation, continuous rotation utilizing well-designed active NiTi files requires less inward pressure and improves hauling capacity auguring debris out of a canal. [3]

On the other hand, a mechanical reciprocating movement has merit because it somewhat mimics manual movement and reduces the various risks associated with continuously rotating a file through canal curvatures. [3]

It has been shown that a single-file reciprocating shaping technique utilizing unequal $\mathrm{CW} / \mathrm{CCW}$ angles is over 4 times safer and almost 3 times faster than using multiple rotary files to achieve the same final shape. [3]

\section{Conclusion}

It is concluded that One Shape and WaveOne, are the new asset in endodontic instruments. Only one rotary file is there for your endodontic treatments. A root canal treatment is approximately 4 times faster than a conventional treatment. Thus the overall duration of treatment is shortened.

\section{References:}

[1]. GhassanYared :Canal preparation with only one reciprocating instrument without prior hand filling : A new concept. Yared 2010

[2]. International Academy for Rotary Endodontics: Single file rotary endodontics - What is new ? Does it make sense?

[3]. Clifford J. Ruddle, DDS: Endodontic canal preparation :WaveOne single-file technique. Dentistry Today, January 2012

[4]. ElioBerutti et al : Root canal anatomy preservation of WaveOne reciprocating files with or without glide path. JOE - Volume, Number 1, January 2012.

[5]. Micro Mega,mmb@micro-mega. com: One Shape : Complete canal shaping with only one single file in continuous rotation.

[6]. Micro Mega, mmid @ micro-mega. com : MM - ENDO book, Your Endo guide. ISO 13485 : \{2004 Certification.\}

[7]. Micro-Mega, Besanc, on, France: One Shape. 2012 